\title{
Vers le naturalisme. La comédie Der G'wissenswurm de Ludwig Anzengruber
}

Unterwegs zum Naturalismus - das Lustspiel Der G'wissenswurm von Ludwig Anzengruber

On the Way to Naturalism - the Comedy Der G'wissenswurm by Ludwig Anzengruber

\section{Sigurd Paul Scheichl}

\section{(Q) OpenEdition}

\section{Journals}

Édition électronique

URL : http://journals.openedition.org/austriaca/494

DOI : 10.4000 /austriaca.494

ISSN : 2729-0603

\section{Éditeur}

Presses universitaires de Rouen et du Havre

\section{Édition imprimée}

Date de publication : 1 juin 2018

Pagination : 25-37

ISBN : 979-10-240-1233-9

ISSN : 0396-4590

\section{Référence électronique}

Sigurd Paul Scheichl, «Vers le naturalisme. La comédie Der G'wissenswurm de Ludwig Anzengruber », Austriaca [En ligne], 86 | 2018, mis en ligne le 01 juillet 2020, consulté le 28 janvier 2021. URL : http:// journals.openedition.org/austriaca/494 ; DOI : https://doi.org/10.4000/austriaca.494 
Sigurd Paul Scheichl

Université d'Innsbruck

\section{Vers le naturalisme}

\section{LA COMÉdie DER G'WISSENSWURM DE LUDWIg ANZENGRUBER}

L'absence du naturalisme serait caractéristique de la littérature autrichienne et une preuve de son indépendance totale à l'égard de la littérature allemande, dans laquelle le naturalisme a joué un rôle important. On retrouve cette idée dans nombre d'études sur l'histoire de la littérature en Autriche, surtout dans celles parues après 1945. La réalité est plus compliquée. Ce mouvement n'est pas du tout absent de la création littéraire autrichienne, même si les histoires de la littérature en Autriche n'en parlent pas ${ }^{1}$. D'un côté, on s'est beaucoup intéressé aux naturalistes européens, on a discuté Zola ${ }^{2}$, on a joué Ibsen et Hauptmann, on a même imité les formes d'organisation des naturalistes berlinois, telle la Freie Bühne. Karl Kraus n'était pas le seul à être enthousiasmé par Hauptmann à Vienne, sans pour autant écrire dans son style. D'un autre côté, un certain nombre d'auteurs autrichiens ont suivi les modèles français, allemands, etc., au moins dans une partie de leur œuvre: Jakob Julius David, Philipp Langmann, Franz Schamann, Felix Salten, Marie Eugenie delle Grazie et d'autres, même Hermann Bahr à ses débuts. Mais il est vrai qu'il n'y a pas eu de mouvement ou de groupe naturaliste en Autriche, bien que des écrivaines et des écrivains aient suivi le modèle naturaliste ${ }^{3}$.

1. Voir Werner Michler, «Zur Frage eines österreichischen Naturalismus», dans Roland Innerhofer et Daniela Strigl (dir.), Sonderweg in Schwarzgelb? Auf der Suche nach einem österreichischen Naturalismus in der Literatur, Innsbruck, StudienVerlag, 2016, p. 21-33., ici p. 21, note 3.

2. Voir Karl Zieger, Die Aufnahme der Werke von Emile Zola durch die österreichische Literaturkritik der Jahrhundertwende, Bern, Lang, «Europäische Hochschulschriften 18,44 ", 1986.

3. Sur les conditions particulières d'un naturalisme en Autriche, voir les observations très intéressantes de Johann Sonnleitner, "Ludwig Anzengruber - Naturalist post mortem? » dans Sonderweg in Schwarzgelb?, op. cit., p. 126-138, ici p. 126 et suiv. 
Il ne faut pas oublier non plus le «naturalisme rural» des Franz Kranewitter et Karl Schönherr ${ }^{4}$, dont les drames se situent dans un milieu paysan et le traitent comme les naturalistes parisiens et berlinois ont représenté le monde de l'industrie, en y découvrant des relations humaines très inhumaines. La critique contemporaine était d'ailleurs tout à fait consciente de la présence du naturalisme en Autriche ${ }^{5}$. Mais, en effet, parmi les auteurs énumérés, l'on ne trouve pas de grands noms, il n'y a pas de Gerhart Hauptmann autrichien; c'est pourquoi il est facile de les oublier et de constater ainsi l'absence de naturalisme en Autriche. En réalité, cette absence n'est qu'une absence du canon littéraire autrichien. Néanmoins, un auteur réaliste autrichien, longtemps populaire, peu connu et encore moins joué aujourd'hui, a même intéressé les naturalistes berlinois, et pour cause: il s'agit de Ludwig Anzengruber (1839-1889) ${ }^{6}$. Ses pièces traitent des problèmes sociaux actuels, surtout la plus célèbre d'entre elles, Das vierte Gebot (Le Quatrième Commandement, 1878). Le sujet en est le déclin de l'artisanat dans les villes et ses conséquences morales: l'alcoolisme, la prostitution, même un meurtre. En 1890, après la mort du dramaturge, la Freie Bühne (le «Théâtre libre» des naturalistes berlinois) a monté et présenté cette tragédie comme texte allemand préfigurant le naturalisme international. Anzengruber a été fait, pour ainsi dire, naturaliste après sa mort ${ }^{7}$.

Si Das vierte Gebot est sans aucun doute proche du naturalisme, cette parenté est moins évidente pour les autres drames de l'auteur dont l'action se déroule souvent dans des villages. Ici, nous proposons une

4. Voir Sigurd Paul Scheichl, «Un naturalisme rural: Franz Kranewitter et Karl Schönherr», dans Karl Zieger et Amos Fergombé (dir.), Théâtre naturaliste: théâtre moderne ? Éléments d'une dramaturgie naturaliste au tournant du XIX ${ }^{e}$ et du $\mathrm{Xx}^{e}$ siècle, Valenciennes, Presses universitaires de Valenciennes, "Études Valenciennoises, 6 ", 2001, p. 79-89; Werner Michler (art. cité, p. 27) souligne aussi les relations de ces auteurs de province au naturalisme. Ailleurs il emploie, non sans ironie, le terme Bauernnaturalismus à propos de Schönherr. Voir Werner Michler, Darwinismus und Literatur. Naturwissenschaftliche und literarische. Intelligenz in Österreich, 1859-1914, Wien, Böhlau, «Literaturgeschichte in Studien und Quellen 2 », 1999, p. 265.

5. Voir quelques jugements sur Kranewitter cités dans Sigurd Paul Scheichl, «Un naturalisme rural », art. cité, p. 80.

6. Pour une bonne introduction, très condensée, à l'œuvre de Anzengruber, voir Karlheinz Rossbacher, "Ludwig Anzengruber (1839-1889)", Nestroyana, $\mathrm{n}^{\circ} 29$, 2009, p. 133-143.

7. Voir Johann Sonnleitner, «Ludwig Anzengruber», art. cité. 
analyse de la comédie Der G'wissenswurm (Le Ver du remords, 1874), qui transpose le Tartuffe de Molière dans un milieu paysan autrichien.

Comme les lecteurs d'Anzengruber sont devenus rares, nous commençons par un bref résumé de l'action. Le riche paysan Grillhofer, veuf depuis quelques années, est devenu dépressif après une maladie. Son beau-frère Dusterer, caractérisé déjà par son nom ${ }^{8}$, essaie de profiter des problèmes psychiques de Grillhofer en expliquant sa maladie selon des doctrines religieuses: elle serait l'effet d'un ver qui rongerait sa conscience (le G'wissenswurm) à cause d'une liaison avec une servante vingt ans auparavant; cette femme serait morte et sûrement en enfer maintenant. Dusterer, le Tartuffe de cette comédie, réussit à convaincre Grillhofer, qui n'a pas d'enfants, que sa seule chance d'échapper à la condamnation au Jugement dernier est d'abandonner ses biens terrestres en les laissant aux pauvres, c'est-à-dire à la famille Dusterer.

L'atmosphère sombre change brièvement lors de la visite d'une jeune femme arrivant d'un village voisin. Il s'agit de la Horlacher-Lies, qui est inconnue du paysan, a beaucoup de tempérament et sait ce qu'elle veut. Ce qu'elle ne sait pas exactement en revanche, c'est la raison pour laquelle sa belle-mère lui a demandé de rendre visite à Grillhofer et lui a conseillé de le flatter. Celui-ci trouve cette jeune femme gaie et très sympathique. Elle repart après quelques heures, mais elle aura quand même appris que Grillhofer et Dusterer vont se rendre en ville le lendemain pour signer l'acte notarial de cession.

Il se passe alors deux choses: Grillhofer apprend par hasard que son ancienne maîtresse est en vie et habite une ferme de la région et que Dusterer s'est bien gardé de l'en informer. Grillhofer se rend tout de suite à la ferme dont le propriétaire a épousé cette Magdalena Riesler. La rencontre avec elle est fort désagréable, mais il apprend qu'elle a eu un enfant dont il est le père, mais qu'elle a abandonné. Peu après, la Horlacher-Lies revient chez Grillhofer pour l'empêcher de céder sa ferme à Dusterer, car, en attendant, sa belle-mère l'a informée qu'elle est la fille du riche paysan. Celui-ci est plus que content de découvrir cet enfant. C'est avec elle et son futur mari qu' il ira chez le notaire pour céder la ferme. Le pieux Dusterer n'a qu'à rentrer chez lui.

Le fait que Der G'wissenswurm soit une comédie n'exclut pas la présence d'éléments naturalistes; il existe d'autres comédies naturalistes,

8. Duster est une forme dialectale de düster, «sombre». Sur le choix des noms, voir Dissertation, Tübingen 1928, p. 69 et suiv. 
dont Der Biberpelz (1892-1893) de Gerhart Hauptmann est probablement la plus célèbre. En tant que comédie, Der G'wissenswurm est tout à fait réussie; Dusterer, surtout, fait rire le public par sa façon de parler comme un curé. Son mot favori est beispielmäßig (par exemple), qu'il répète sans cesse pour convaincre Grillhofer de ses péchés. Anzengruber choisit un mot qui n'est pas courant ${ }^{9}$ - et qui appartient à l'allemand littéraire plutôt qu'au patois - pour mettre encore plus en relief le mauvais caractère du Tartuffe de la pièce et pour le rapprocher de la langue du clergé (que l'auteur n'aimait pas du tout). Grillhofer fait même une comparaison entre son beau-frère et un curé: «Der Dusterer is über ein Feldpater» (Dusterer est meilleur qu'un curé militaire, I, 4$)^{10}$. Et il continue par une ambiguïté voulue par l'auteur : «A Teuxelskerl [...] mit sein' gottg'fälligen Wesen» (Un type formidable et si agréable à Dieu). Le jeu de mots ne peut se traduire: Teuxelskerl (patois pour Teufelskerl), un mot qui exprime l'admiration, contient quand même le mot Teufel (diable). Sans le vouloir, Grillhofer caractérise le pieux Dusterer, qui a bien un côté diabolique, par le mot Teuxelskerl, qui anticipe ainsi les événements. Ce détail linguistique montre, comme beaucoup d'autres, qu'Anzengruber a travaillé très scrupuleusement son texte.

D’autres éléments comiques ne manquent pas. Au début, Grillhofer ne cesse de se plaindre de la mauvaise journée et mange sa soupe en disant qu'elle ne lui servira à rien, alors que, selon les indications scéniques, il coupe le pain rapidement et boit la soupe avec avidité ( "mit Gier», I, 2). Le comportement du mari et des fils de Magdalena Riesler, quand ils essaient d'échapper au régime de la paysanne (II, 11 à 14), serait un autre exemple de ces effets comiques. La répétition de beispielmäßig (vingt et une fois, dont une dans la bouche de Liesl, une dans celle de Grillhofer) n'est pas le moindre de ces effets. Anzengruber sait construire une pièce qui fait rire le public.

La tradition dans laquelle la pièce s'insère par ce genre de motifs est celle de la comédie populaire, du Volksstück autrichien, plus exactement celle de la comédie qui se déroule dans un milieu paysan. Anzengruber

9. Le mot beispielmäßig ne se trouve ni dans le Deutsches Wörterbuch ni dans des dictionnaires récents. On dirait plutôt beispielsweise.

10. Nous donnons l'acte et la scène pour que l'on puisse retrouver les passages facilement dans toutes les éditions. Le texte est cité d'après Ludwig Anzengruber, Ausgewählte Werke in vier Bänden, Carl Wilhelm Neumann (éd.), Leipzig, Reclam, 1928, t. IV, p. 197-250. Le texte de la $1^{\text {re }}$ édition est disponible à l'adresse suivante: http://www.deutschestextarchiv.de/book/show/ anzengruber_gwissenswurm_1874 (consultation le 5 novembre 2018). 
n'avait pas de programme innovateur quand il a écrit cette Bauernkomödie (comédie paysanne), mais il voulait surtout réussir. (Ce qui pouvait être provocateur, c'étaient les motifs anticléricaux, mais non des innovations formelles.) Bien que le mot «naturaliste» ait été utilisé par Zola dès 1868 , il est d'ailleurs peu probable que le naturalisme en tant que méthode littéraire ait déjà fait l'objet de discussions dans les milieux littéraires viennois à l'époque où Anzengruber a écrit Der G'wissenswurm (en 1874). Avant Der G'wissenswurm, Anzengruber avait déjà réussi avec d'autres pièces de ce genre, dont Der Pfarrer von Kirchfeld (Le Curé de Kirchfeld, 1870) et Die Kreuzelschreiber (Les Analphabètes, 1872).

Deux éléments surtout sont l'héritage du Volksstück: le dialogue, qui est écrit dans le patois du sud des pays de langue allemande ${ }^{11}$, et l'importance de la musique. Anzengruber lui-même appelle sa pièce Bauernkomödie mit Gesang (comédie paysanne avec chants). Mais son emploi des chansons n'est pas du tout naif; la concession à la tradition et aux effets de théâtre est utilisée habilement pour souligner le contraste entre le monde triste de Dusterer et de Grillhofer, empreint de cléricalisme, et la vitalité de Horlacher-Lies et de son amant. En I, 11, Anzengruber en tire un effet comique dont la fonction critique n'est pas négligeable. La scène finit par une chanson de la Horlacher-Lies reprise par le chœur et culminant dans un Jodler, une mélodie sans texte caractéristique de la tradition musicale des Alpes:
O schön' grüne Welt,
Laß sag'n, wie d'mer g'fallst,
Solang Zithern klingen
Und mei Dirndl mich halst ${ }^{12}$.

Quand le chœur a fini, on entend de nouveau le chant de pénitence (Bußlied) pieux que Dusterer a commencé à chanter avec sa victime dès la fin de I, $8^{13}$. Le Gesang, la musique, est ici beaucoup plus qu'un effet de scène traditionnel: les deux chansons marquent le contraste

11. Anzengruber n'emploie pas le dialecte d'une région précise pour rendre possible une adaptation facile au lieu d'une mise en scène. Voir Karlheinz Rossbacher, art. cité, p. 135.

12. "Mon beau monde vert, laisse-moi te dire comme tu me plais, tant que j'entends les cithares et que ma petite amie m'embrasse.» La Zither est l'un des instruments les plus répandus de la musique populaire des pays alpins.

13. Il se peut que le texte de Anzengruber soit une parodie ; s'il cite un texte religieux, il en a choisi un qui se rapproche du ridicule, surtout avec la rime "Gnaden/schaden" (grâce/nuire). 
central de cette comédie, le contraste entre vie et vitalité d'un côté et une piété sombre, hostile à la vie, de l'autre. Le fait que Liesl chante dans les prés avec les ouvriers de la ferme, tandis que l'on entend Grillhofer et Dusterer derrière la scène (les deux agissent d'ailleurs pratiquement toujours à l'intérieur de la ferme), accentue cet effet. Que les mots de Liesl soient repris par un chœur et soient donc plus forts que le chant des deux vieux hommes souligne la confrontation. L'importance de cette chanson gaie et le triomphe de l'attitude qu'elle exprime sont soulignés par le fait que Grillhofer la reprend juste avant la fin de la pièce, en III, 7.

Jouer ainsi avec la musique n'est évidemment pas une technique propre au naturalisme, mais le but de cette confrontation préfigure les thèmes de celui-ci. Anzengruber, figure de proue de l'anticléricalisme en Autriche (pays très catholique à l'époque), dépasse ici la simple critique de l'Église et juxtapose l'amour de la vie aux règlements moraux traditionnels. Le péché commis par Grillhofer des années auparavant ne paraît pas comme tel, mais comme quelque chose de naturel : la religion, ennemie de la sexualité, est présentée comme élément destructeur, comme méthode visant à supprimer les hommes. Ce n'est pas par hasard qu'Anzengruber fait comparer, la vie chez Grillhofer à la vie dans une chartreuse par le garçon de ferme Wastl : « [...] daß mir hitzt dasitzen wie aufeiner Kartausen $(\mathrm{I}, 4)^{14}$. Il faut remarquer que l'auteur attribue cette réplique à un personnage dont l'éducation rend plutôt invraisemblable qu'il soit informé sur l'ordre des chartreux, disparu en Europe centrale depuis le $\mathrm{XVIII}^{\mathrm{e}}$ siècle, et sur ses règles très strictes.

Cette position à l'égard de la question du péché et cette réserve envers la religion ne font pas de Der G'wissenswurm une pièce naturaliste, mais la comédie montre ainsi que le monde des années 1870 était mûr pour le naturalisme. Bien entendu, les dramaturges de cette école ne se serviront plus de chansons, ni pour amuser le public ni pour souligner les contrastes entre la réalité de la vie et des principes moraux abstraits. Mais ces contrastes continuent à les intéresser.

L'emploi du patois dans les dialogues se retrouvera dans les œuvres naturalistes, dans lesquelles il aura une autre fonction. Chez Anzengruber, c'est un moyen de se rapprocher de la langue de son public qui, même à Vienne, ne parlait pas l'allemand littéraire. Pour le naturalisme, l'emploi $\mathrm{du}$ patois ou d'une langue relativement vulgaire sera exclusivement un moyen de donner une image précise du milieu dans lequel se

14. "Que nous nous comportons maintenant comme dans une chartreuse.» 
déroule l'action. Ces intentions différentes n'excluent pas que certains naturalistes de langue allemande aient emprunté cette technique du dialogue au Volksstück.

Examinons maintenant les sujets de la comédie. Le sort de la HorlacherLies, caractère fort sympathique, fait partie des motifs prénaturalistes de Der G'wissenswurm. En 1874, peu d'auteurs ont osé choisir un enfant illégitime comme protagoniste d'un roman ou d'un drame. Anzengruber parle très clairement du sort que la société de l'époque (sous l'influence de l'Église) réservait très souvent à ces enfants. La mère, n'ayant ni le temps ni l'argent pour s'occuper de son bébé, ne pouvait faire autre chose que de l'abandonner, même si Magdalena a réussi à extorquer de l'argent à la femme de Grillhofer. Mais ensuite, elle ne sait même pas où sa fille se trouve et ce qu'elle est devenue (II, 15). Le public devine déjà que cette fille a eu de la chance en trouvant une bellemère qui s'est bien occupée d'elle: en effet, on soupçonne très tôt que cet enfant abandonné est la Horlacher-Lies. La critique sociale (et anticléricale) est ainsi mitigée, mais elle reste impliquée dans l'action de Der G'wissenswurm. Néanmoins, si l'histoire de la fille de Grillhofer renvoie à un problème qui intéressera le naturalisme, le personnage assez harmonieux de la jeune femme est caractérisé d'une façon qui a peu en commun avec les personnages des drames naturalistes. Il faut noter que les naissances illégitimes dans les villages étaient très nombreuses tout au long du XIX ${ }^{\mathrm{e}}$ siècle, malgré la position forte de l'Église.

Dans la scène où Grillhofer retrouve la mère de son enfant, il est aussi question de sexualité sous un autre aspect. Son ancienne maîtresse qui, devenue hargneuse, veut bien entendu humilier son ancien amant, lui dit brutalement que l'affaire d'antan était calculée de sa part : elle avait pensé que la femme de Grillhofer, fréquemment malade, allait bientôt mourir et qu'elle, Magdalena, allait remplacer la riche paysanne (II, 15) : «Net a so viel (schlägt ein Schnippchen), sixt, war mer sunst an dir g'leg'n $n^{15}$.» Ce genre d'ambition, qui pourrait correspondre à la réalité du monde agricole, réapparaît dans d'autres œuvres d'Anzengruber.

Avant de retourner à la scène avec Magdalena, nous souhaiterions attirer l'attention sur un autre aspect problématique de la vie dans les villages, dont il est question dans les dialogues. À plusieurs reprises on

15. «Autrement, tu m'aurais si peu intéressée - elle fait claquer ses doigts. » 
parle de la Ausnahm ${ }^{\prime 16}$ (I, 4 et 8 ; II, 4; III, 7). Il s'agit d'un règlement plutôt social, voire sociétal, que légal de la vie des anciens propriétaires de fermes: une fois la ferme cédée, le fils héritier était obligé de loger et de nourrir ses parents jusqu'à la fin de leurs jours. Au moins une fois Grillhofer pense à des circonstances bien connues jusqu'au $\mathrm{xx}^{\mathrm{e}}$ siècle: "Aber in d'Ausnahm' gehn, wo andere mit ihnere leibliche Kinder aften nix Gut's derleb'n, zu Fremde auf Gnoden und Ungnoden!?» $(\mathrm{I}, 8)^{17}$. Très souvent en effet, la propriété ne rapportait pas assez et les héritiers s'occupaient trop peu des parents. En faisant évoquer par l'intermédiaire de Grillhofer un problème social grave, Anzengruber est ici loin de toute idéalisation de la vie des paysans ${ }^{18}$. Quand le protagoniste, dans son avant-dernière réplique, semble, avec un jeu de mots, tout content de la Ausnehmerei imminente chez Liesl et Wastl, l'auteur s'éloigne de l'image pré-naturaliste du monde paysan : la comédie l'emporte alors sur le réalisme.

Revenons à présent aux scènes II, 11 à 16 qui se passent à la ferme de Magdalena, dont le seul nom, Kahle Lehnten (pente dénudée), indique déjà qu'il s'agit de terrains peu fertiles et que la famille Poltner est donc plutôt pauvre. En effet, leur ferme est située aux pieds de la montagne. Le paysan est décrit comme quelqu'un qui se néglige ("auch im übrigen Anzuge zeigt sich eine arge Vernachlässigung », II, 11) et, de plus, il boit, bien que sa femme ne lui donne pas d'argent pour aller au village. Il obtient la somme nécessaire de ses fils qui en profitent pour échapper ainsi au contrôle paternel et avoir de petites amourettes.

La description du décor matérialise déjà les contrastes existant dans le milieu agricole. La longueur même des didascalies indique qu'elles n'ont pas une simple fonction d'organisation spatiale de ce qui se passera, mais qu'ils donnent des informations sur le milieu (ce changement de fonction des indications scéniques se trouvera aussi dans les pièces naturalistes). Alors que la grande ferme de Grillhofer permet évidemment

16. Aujourd'hui le mot correct serait Ausgedinge ou Altenteil. Le mot Ausnahme, dans ce sens, ne se trouve ni dans le Deutsches Wörterbuch ni dans des dictionnaires plus récents. En raison des évolutions sociales, Ausgedinge n'est plus un mot allemand courant.

17. "Mais est-ce que c'est une bonne idée que de se retirer de la ferme et de dépendre totalement d'autrui quand bien des gens font de mauvaises expériences avec leurs propres enfants?»

18. Johann Sonnleitner, "Ludwig Anzengruber», art. cité, p. 133, souligne qu'Anzengruber était bien au courant des conditions économiques et sociales dans les villages. 
une vie aisée à son propriétaire, la maison de Magdalena Poltner est beaucoup plus modeste (voir le début de II, 11). L'entrée dans cette petite ferme est ouverte, il n'y a pas de clôture qui protégerait l'ensemble des bâtiments, tandis que le jardin de Grillhofer est fermé par une barrière faite dans un bois de bonne qualité ( aus Prügelholz genagelten Einlaßschranken », II, 1). L'auteur nous informe aussi que les montagnes s'élèvent directement derrière cette "pente dénudée "; par contre, de la ferme de Grillhofer, les Alpes ne se voient qu'au loin, en arrière-plan (II, 1). Les particularités de l'extérieur des fermes n'ont pas d'incidence sur les événements, elles servent uniquement à établir le contraste entre paysan pauvre et paysan riche. D'autres détails montrent que les Poltner - avec leurs douze enfants - ne sont pas à l'aise; ils ne savent même pas lire. Leurs moyens ne leur permettraient pas de prodiguer l'hospitalité que le paysan aisé offre généreusement à tous ceux qui viennent.

En II, 12, la Bäuerin, Magdalena, donne l'ordre à son mari et à ses grands fils de tricoter pour les enfants plus jeunes, une scène qui avait certainement un effet très comique en 1874 , car un homme ne tricotait pas. Mais cet ordre sert aussi à caractériser la paysanne en tant que personne très dominante et à nous présenter la pauvreté de la famille. Les lectrices et les spectatrices sont invitées à s'expliquer le contraste entre la Magdalena de maintenant et la jeune femme attrayante d'antan (que nous rappelle sa fille si sympathique) par sa triste biographie - un autre élément de la critique sociale fort présente dans cette comédie. Que nous puissions être amenés à penser que Grillhofer a peut-être eu de la chance en n'épousant pas cette femme est un motif comique qui n'a rien à voir avec le réalisme critique de Der G'wissenswurm.

Les scènes à la ferme Kahle Lehnten ont ainsi plusieurs fonctions dont l'une des plus importantes est celle de contraster les milieux. Aussi peu aimable que soit Magdalena, sa dure existence lui donne quand même quelques excuses. De Dusterer, nous apprenons qu'il n'a pas beaucoup d'argent, mais il ne nous est pas présenté dans son milieu, ce qui pourrait le rendre un peu plus antipathique. Le contraste des milieux et leur influence sur le comportement des personnages sont importants dans cette comédie qui a bien des traits d'une analyse sociale et dépasse ainsi les limites du Volksstück traditionnel.

Nous donnerons ici juste un exemple des qualités esthétiques de la pièce: Dusterer a stimulé la mauvaise conscience de Grillhofer, en lui racontant que son ancienne maîtresse serait en enfer, il l'y aurait vue dans ses rêves. Quand Grillhofer apprend que Magdalena est bien en vie et habite à trois heures de voyage de sa ferme, il se rend compte que son 
beau-frère a menti : "Erzlugner! Is die Höll' a drei Stund' von da an der kahlen Lehnten? Is dort die Höll', Erzlugner?» (II, 8) ${ }^{19}$. Cette question rhétorique s'avère très réaliste, quand la vie dans cette ferme pauvre nous est présentée. Anzengruber est ici bien plus ironique que le Volksstück ne l'est normalement.

L'anticléricalisme est aussi un élément de cette analyse d'une société dominée par l'Église et la superstition. Bien sûr, Dusterer est un hypocrite et ses arguments ne sont pas ceux de l'Église, mais il sait profiter des opinions basées sur les doctrines catholiques relatives à l'enfer et au purgatoire, qu'il interprète de la façon qui l'avantage. Le fait que les péchés dans le domaine sexuel (pour Anzengruber, naturel) soient si importants pour les paysans pieux que Dusterer puisse en profiter, est davantage qu'un élément de comédie; il fait partie, dans Der G’wissenswurm, de la critique de la société autrichienne.

Le contraste entre la chanson de contrition, que Dusterer fait chanter à Grillhofer, et la chanson de Liesl sur le beau monde exprime un contraste important pour Anzengruber: il est au fond plus qu'anticlérical: sa conception du monde est plutôt celle des sciences que celle de la théologie. C'est très clair encore lors de la deuxième rencontre entre Grillhofer et sa fille: lui, après la visite chez Magdalena, ne pense qu'au cimetière, tandis qu'elle ne parle que de la beauté de la nuit et du ciel clair; elle a l'impression que son âme est tirée de son corps et fait partie du ciel lumineux (III, 6). Anzengruber s'approche ici du panthéisme ${ }^{20}$.

C'est pourquoi il ose même ridiculiser l'enfer, quand il fait raconter à Dusterer ses rêves habités par Magdalena qui se trouverait dans le feu éternel de l'enfer ou peut-être au purgatoire (I, 8). Et quand on apprend que Magdalena est en vie, Dusterer explique que l'on voit mal à cause de la fumée et qu'il a donc pu confondre Magdalena avec son enfant, qui ressemble à sa mère et qui se trouverait au purgatoire (III, 3 ). Cette image naïve des peines éternelles et le commentaire sarcastique de Grillhofer : "Weil dir's taugt, steckst dös hitzt ins Fegefeuer» (III, 3$)^{21}$ font rire le

19. «Fieffé menteur! Est-ce que l'enfer est à trois heures d'ici à la kahlen Lehnten? Est-ce que l'enfer est là-bas, fieffé menteur?"

20. Ce sont surtout ces prises de position libérales qui ont fait disparaître Anzengruber, comme maints autres, du canon autrichien dans un processus de réception compliqué. Voir Sigurd Paul Scheichl, «Bissige Literatur - zahnloser Kanon. Zu Fragen der literarischen Tradition in Österreich », Sprachkunst, ${ }^{\circ}{ }^{28}$, 1997, p. 247-274. En effet, depuis 1945 il y a eu très peu d'éditions des œuvres d'Anzengruber en Autriche ; la seule édition sérieuse a paru en RDA (Aufbau Verlag) en 1971.

21. «Parce que cela t'arrange, tu la bannis maintenant au purgatoire. » 
public, mais ils ont certainement déplu à la censure autrichienne des années 1870 , qui n'appréciait pas du tout des positions contraires aux doctrines catholiques.

En résumé, Der G'wissenswurm n'est certainement pas une pièce naturaliste, en tout cas bien moins que ne le sera Das vierte Gebot quatre ans plus $\operatorname{tard}^{22}$. Anzengruber y reste dans la tradition du Volksstück, de la comédie populaire, qui d'ailleurs promettait des succès de scène et des revenus. Néanmoins, l'auteur savait que ce milieu était en train de changer et, de toute façon, n'était pas aussi idyllique que la Heimatkunst, la littérature du terroir, dont les débuts étaient contemporains, le prétendait. Dusterer, le Tartuffe de Der G'wissenswurm, n'est pas du tout un caractère naturaliste, mais d'autres caractères, surtout dans la famille de Magdalena, ont des traits qui préfigurent ce que nous avons appelé ailleurs un "naturalisme rural $^{23}$ » et dont les premiers exemples datent des années 1890.

Le transfert de Tartuffe dans le milieu paysan alpin est assez loin de la nouvelle théorie littéraire, et l'on ne trouve pas, ou à peine, dans Der G'wissenswurm, les positions théoriques du naturalisme. Anzengruber est loin de faire une expérience avec les caractères de sa comédie. Le milieu, par contre, est dépeint d'une façon qui, parfois, est naturaliste avant le naturalisme (au moins avant le naturalisme allemand), surtout dans les scènes à la Kahle Lehnten et par le contraste entre cette ferme et celle de Grillhofer.

C'est surtout le grand intérêt de l'auteur pour les problèmes du monde agricole qui rapproche Der G'wissenswurm des naturalistes; comme eux, il essaie de les analyser précisément. Parmi ces problèmes, il y a la situation sociale du personnel agricole, la pauvreté de bon nombre de paysans (souvent analphabètes) et le sort des vieux paysans qui ont laissé leurs biens à des héritiers. Ces problèmes sociaux sont quand même présentés dans un contexte plus large. Il est ainsi question de l'influence du milieu sur le sort individuel; Anzengruber ne laisse pas subsister de doute sur le fait que la Magdalena hargneuse de la Kahle Lehnten est devenue si agressive à cause de sa vie dure, et même les intrigues de Dusterer s'expliquent un peu par les conditions de sa vie.

22. Pourtant, les naturalistes berlinois joueront aussi Der G'wissenswurm entre 1893 et 1895 .

23. Sigurd Paul Scheichl, «Un naturalisme rural», art. cité. 
Un autre aspect général traité dans la comédie est l'obsession pour le péché de la sexualité. Nous n'avons pas parlé de l'amour entre Lies et Wastl, le garçon de ferme chez Grillhofer, une relation qui promet de rendre heureux les deux; mais elle est plutôt une concession aux règles de la comédie. Lies, bien sobre, dit à Grillhofer qu'il faut être deux pour une liaison et qu'il n'est donc pas entièrement responsable de son comportement fautif vis-à-vis de la servante (II, 4), mais Dusterer réussit à le convaincre de son péché. Il est évident qu'Anzengruber n'était pas partisan de la théologie morale de l'époque. Sans pouvoir rentrer dans les détails, nous ajouterons quand-même que dans un texte théorique, «Eine Plauderei als Vorrede» (Une causerie en guise de préface), qui introduit le deuxième volume de ses Dorfgänge (Promenades au village) (1879), Anzengruber insiste sur la nécessité de parler des relations sexuelles, si l'on veut être un écrivain réaliste dont le programme est de dire la vérité.

Plusieurs motifs de Der G'wissenswurm montrent donc qu'Anzengruber est sur la voie qui mène au le naturalisme. D'après Sprengel, une grande innovation réussie par l'auteur est le choix du milieu agricole pour l'évocation de problèmes actuels, qui sont importants aussi pour les citadins ${ }^{24}$. Dans ses notes des années 1880, Anzengruber sera encore plus proche du nouveau mouvement ${ }^{25}$ et discutera même les écrits de Zola, mais le chemin qui mène de Der G'wissenswurm à la théorie naturaliste sera encore long ${ }^{26}$. Pourtant, plusieurs motifs et techniques de cette comédie montrent que la littérature était prête pour le naturalisme et justifient, comme d'autres œuvres du dramaturge viennois, de le classer parmi ses prédécesseurs. Néanmoins, la comédie n'est pas une pièce naturaliste dans le sens strict du terme.

Un dernier argument pour rapprocher Anzengruber du naturalisme est sa réception, dans les recherches littéraires comme au théâtre. Une thèse de doctorat de 1928 est intitulée tout simplement: Anzengrubers Naturalismus ${ }^{27}$. Le germaniste berlinois, qui aborde d'ailleurs les

24. Peter Sprengel, Geschichte der deutschsprachigen Literatur 1870-1900, München, Beck, « de Boor/Newald, Geschichte der deutschen Literatur von den Anfängen bis zur Gegenwart IX, 1», 1998, p. 460.

25. Werner Michler, Darwinismus und Literatur, op. cit., p. 200.

26. Peter Sprengel, op. cit., p. 466.

27. Fritz Weber, Anzengrubers Naturalismus, op. cit. Nous n'avons pas pu consulter Karl Ermisch, Anzengruber und der Naturalismus, thèse, University of Minnesota, 1927 (disponible à la Deutsche Nationalbibliothek à Leipzig et à la Bibliothèque nationale de France). 
différences entre l'œuvre d'Anzengruber et celle de certains naturalistes, n'a pas hésité à qualifier l'auteur de naturaliste. Son travail s'appuie sur toutes sortes de remarques théoriques d'Anzengruber - et il se peut effectivement que le dramaturge soit plus près du nouveau mouvement sur le plan de la réflexion que dans ses pièces et contes ${ }^{28}$.

La réception sur scène a, elle aussi, rapproché Anzengruber du naturalisme. D'un côté, nous avons mentionné les productions berlinoises autour de $1890^{29}$, de l'autre, le dramaturge était l'un des auteurs-phare des programmes de la Exl-Bühne $e^{30}$, un ensemble de haut niveau, qui était actif surtout dans le Tyrol et à Vienne, mais qui jouait aussi souvent dans tous les pays de langue allemande, un ensemble spécialisé dans le naturalisme rural des Kranewitter (1860-1938) et Schönherr (1867-1943). Ils ont intégré Anzengruber (né en 1839) à cette tradition en en faisant un ancêtre. Dans la première moitié $\mathrm{du} \mathrm{xx}^{\mathrm{e}}$ siècle, on a souvent pu voir Der G'wissenswurm interprété par les excellents acteurs et actrices de cet ensemble. Voir Eduard Köck dans le rôle de Dusterer - comme lors du Festival de Salzburg en $1943^{31}$ - a dû être une soirée de théâtre inoubliable.

28. Voir Fritz Weber, op. cit., p. 106.

29. Voir la liste dans Werner Michler, Darwinismus und Literatur, op. cit., p. 198.

30. Voir Ekkehart Schmidl, Der Traum vom Volkstheater. Die Geschichte der ExlBühne (1902-1956), Innsbruck, Haymon, «Veröffentlichungen des Innsbrucker Stadtarchivs N. F. 49 ", 2013.

31. La première a eu lieu le 7 août 1943 ; nous n'avons pas pu trouver d'articles sur cette production. 